Techniques \& Culture

\title{
La seconde vie du cæcum
}

ou le stockage ingénieux du beurre en Mongolie

\section{Sandrine Ruhlmann}

\section{(2) OpenEdition}

Journals

Édition électronique

URL : https://journals.openedition.org/tc/8809

DOI : $10.4000 /$ tc. 8809

ISSN : 1952-420X

Éditeur

Éditions de l'EHESS

\section{Édition imprimée}

Date de publication : 17 mai 2018

Pagination : 74-87

ISBN : 978-2-7132-2750-9

ISSN : 0248-6016

Référence électronique

Sandrine Ruhlmann, «La seconde vie du cæcum », Techniques \& Culture [En ligne], 69 | 2018, mis en ligne le 17 mai 2020, consulté le 29 septembre 2022. URL : http://journals.openedition.org/tc/8809 ; DOI : https://doi.org/10.4000/tc.8809 


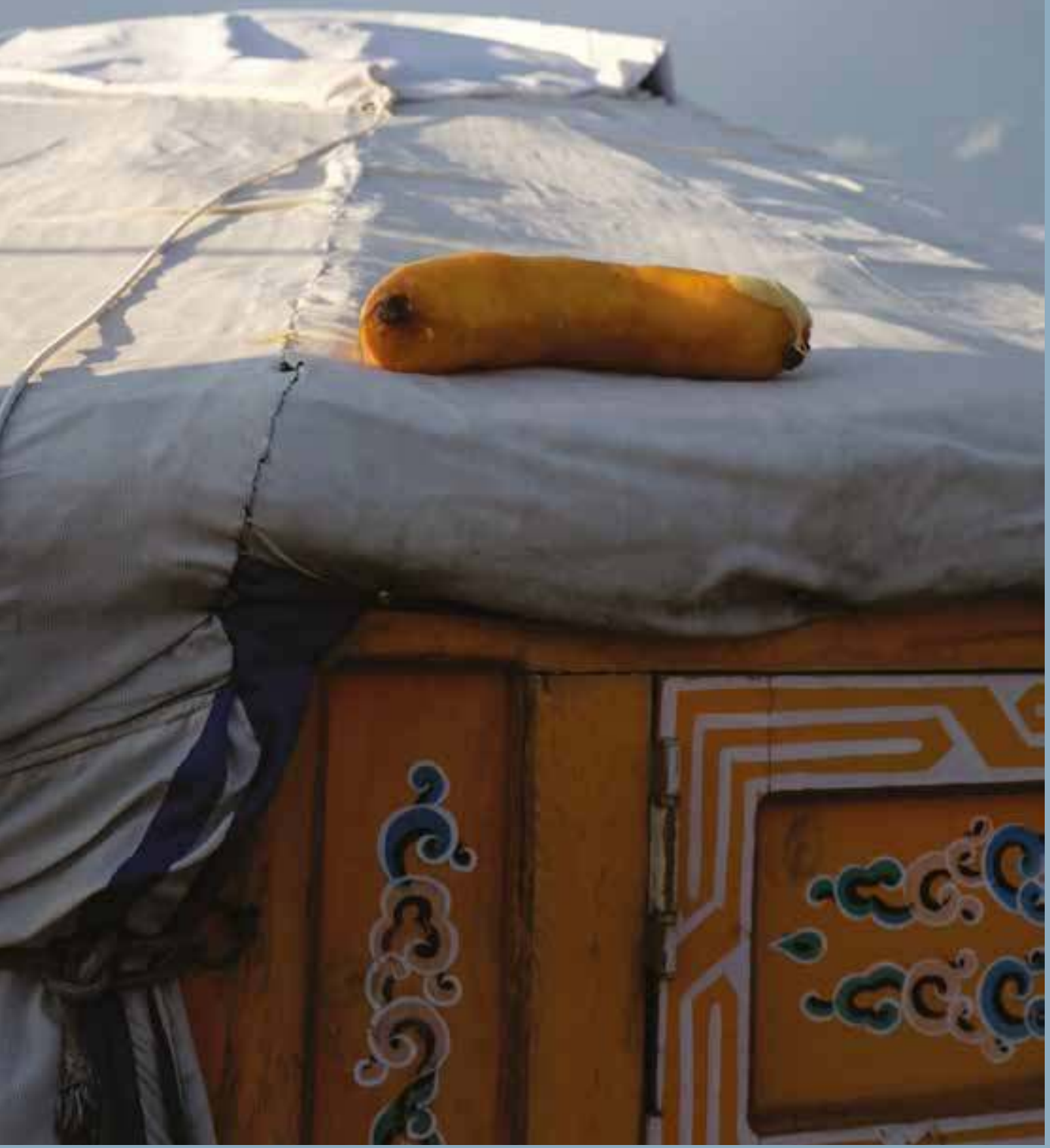




\section{La seconde vie du cæcum ou le stockage ingénieux du beurre en Mongolie}

Soit un contenant, le cæcum de mouton, et un produit qu'il sert à conditionner, stocker et transporter sans encombre, le beurre. La Mongolie est un pays d'Asie septentrionale au climat continental extrême, habité par des familles, dont des éleveurs, nomades ou sédentaires, où la question du stockage des aliments se substitue à celle de leur conservation. La fermentation, ainsi que la dessiccation, la surgélation et le fumage ne sont pas, dans la culture mongole, des techniques de conservation, mais de stockage. Il s'agira ici de s'emparer de deux matières, le boyau et le beurre, comme les femmes le font, engageant leur corps et leurs outils pour les travailler, la fabrication des produits laitiers leur incombant. Fondée en particulier sur la rencontre avec une famille d'éleveurs nomades en 2017 dans la province du Töv, au centre de la Mongolie, et sur des observations menées de 2000 à 2017 dans la province du Hentij, à l'est, cette expérience narrative est une tentative de vous faire sentir l'odeur, la couleur et la texture du beurre mongol, jusqu'à en saisir son goût si particulier.

Selon sa taille, le cæcum peut contenir 3 à 6 kilogrammes de beurre. Les Mongols ajoutent de petites cuillérées de ce beurre, rance à l'issue de sa fabrication, dans différentes préparations culinaires: thé au lait, bouillies de laitages et de céréales, pâtisseries. Certains l'étalent généreusement, comme la peau crémeuse du lait, sur des tranches de pain, saupoudrant le tout de sucre. Pour stocker ce beurre, les éleveurs nomades utilisent des matières organiques animales recyclées, comme le cæcum, une poche de l'appareil digestif du ruminant dont l'éleveur dispose quand il a abattu un animal. Vidé des matières fécales, donc de l'herbe dont l'animal vivant s'est nourri, c'est un contenant peu encombrant, léger, durable et transportable. Sa membrane souple et épaisse présente l'élasticité et l'étanchéité requises pour renfermer, sans risque de fuite ou de déperdition, une quantité de beurre, donc un dérivé d'une substance animale transformée, stocké à l'état naturel mou, liquide (liquéfié) ou dur (congelé) selon la température saisonnière ambiante (de +40 l'été à $-40^{\circ} \mathrm{C}$ l'hiver). 
Plus encore que chez les familles sédentaires, d'éleveurs ou non, chez les éleveurs nomades mongols, qui ne pratiquent pas l'agriculture mais occasionnellement chassent et cueillent, le stockage relatif ${ }^{1}$ et le transport des aliments sont primordiaux, tandis que la conservation au sens de non-altération passe au second plan. Les éleveurs transforment le lait de sorte que les produits dérivés aient un goût, une texture, une odeur et une couleur inaltérables (laitages séchés ou congelés, lait déshydraté) ou que la marge d'altération possible (laitages fermentés ou caillés) soit entièrement désirée. À partir de la traite, chaque étape de fabrication d'un produit laitier découle de la précédente, tel «un seul processus »: chauffage (lait) ${ }^{2}$, écrémage du lait (peau crémeuse, crème), barattage de la crème (beurre), fermentation du lait (yaourt), égouttage/ pressage du yaourt (caillé), séchage du caillé (fromages). «C'est un procédé, on fait la peau crémeuse du lait un jour, du yaourt et du beurre le lendemain, des fromages avec le reste le jour d'après. » L'évolution des qualités olfactive, gustative, tactile et visuelle des produits n'entraîne ni dégoût, ni restriction, ni contre-indication de consommation. Les éleveurs ne se posent pas la question de la conservation et, à ce titre, aucun terme ne désigne la conservation si ce n'est le terme signifiant le stockage (hadgalalt). C'est bien la question du stockage qui importe aux nomades, au sens de réduction en nombre et en volume des possessions matérielles pour faciliter leur transport lors des nomadisations ou autres déplacements.

La fabrication estivale du beurre rance permet de constituer, dès la fin de l'automne, des provisions «de gras nourrissant». J'ajouterais que les familles disposeront ainsi de protéines, d'acides aminés et de vitamine A durant les mois d'hiver rigoureux. L'altération du goût, de la texture, de l'aspect et de l'odeur n'est pas préoccupante; elle est même pour la peau crémeuse du lait (öröm) une évolution souhaitée, telle une maturation spontanée, hors processus, ce d'autant plus que sa version fermentée (peau crémeuse fermentée, zööhij) est, généralement, le produit de base de fabrication du beurre. Stocké jusqu'à épuisement, le beurre garde une texture « onctueuse», sa couleur «jaune vif» et son goût «fort» tant prisés: «faire du beurre, c'est laisser rancir la peau crémeuse du lait, baratter et stocker ». Lorsque le goût convoité est obtenu, le produit est «stocké longtemps», plusieurs mois, une année, dans son contenant rangé dans l'entrepôt extérieur, bon pour la consommation sans date de péremption. Les familles peuvent le transporter à la main ou en tirant l'entrepôt mobile, monté sur roulettes. 


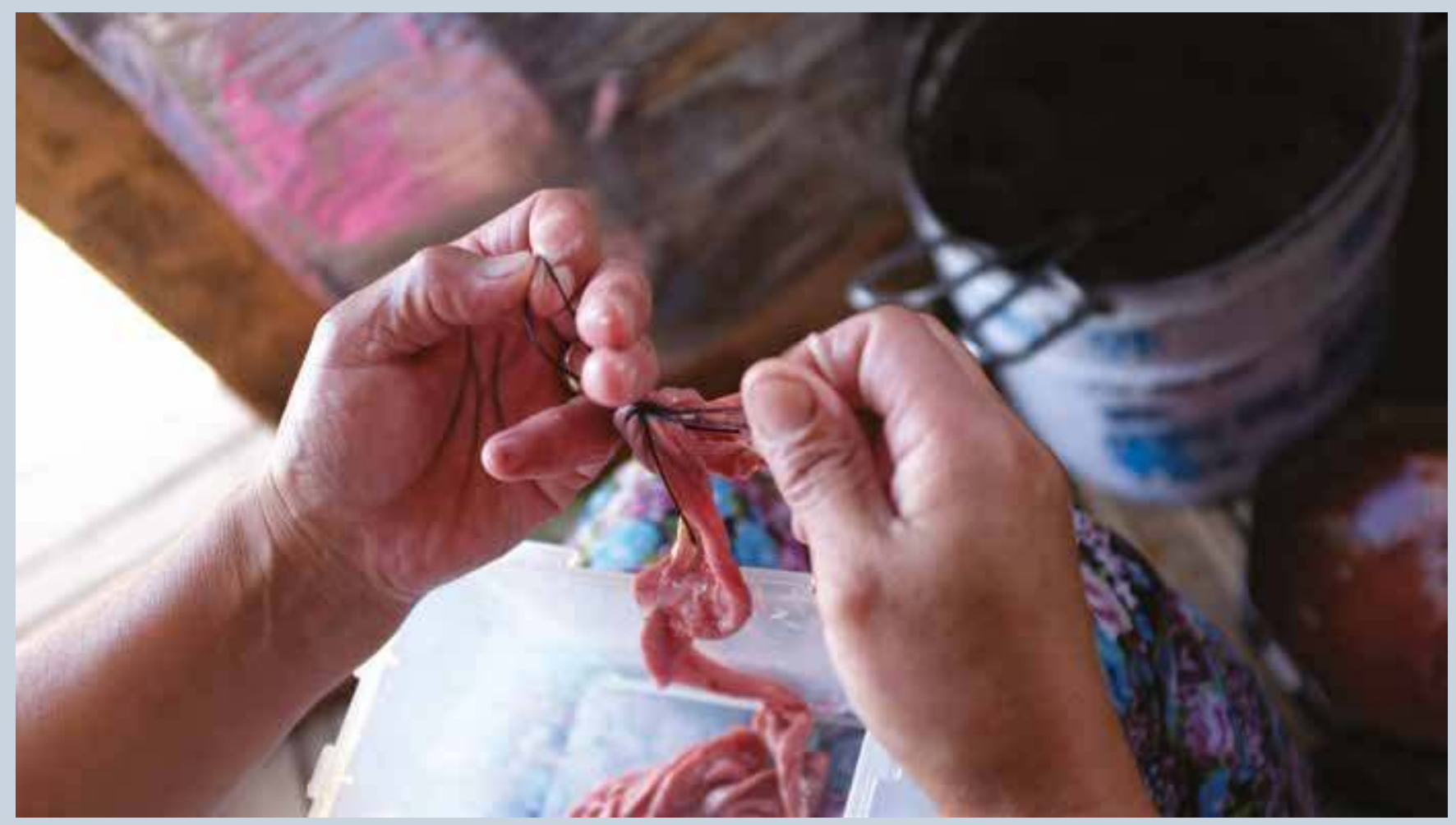

1. La maîtresse de maison lave minutieusement les parois interne et externe de la membrane avec de l'eau, ici non mêlée de lait. Elle ôte délicatement certaines des adhérences sur la paroi externe, pour s'assurer de la bonne élasticité de la membrane. 


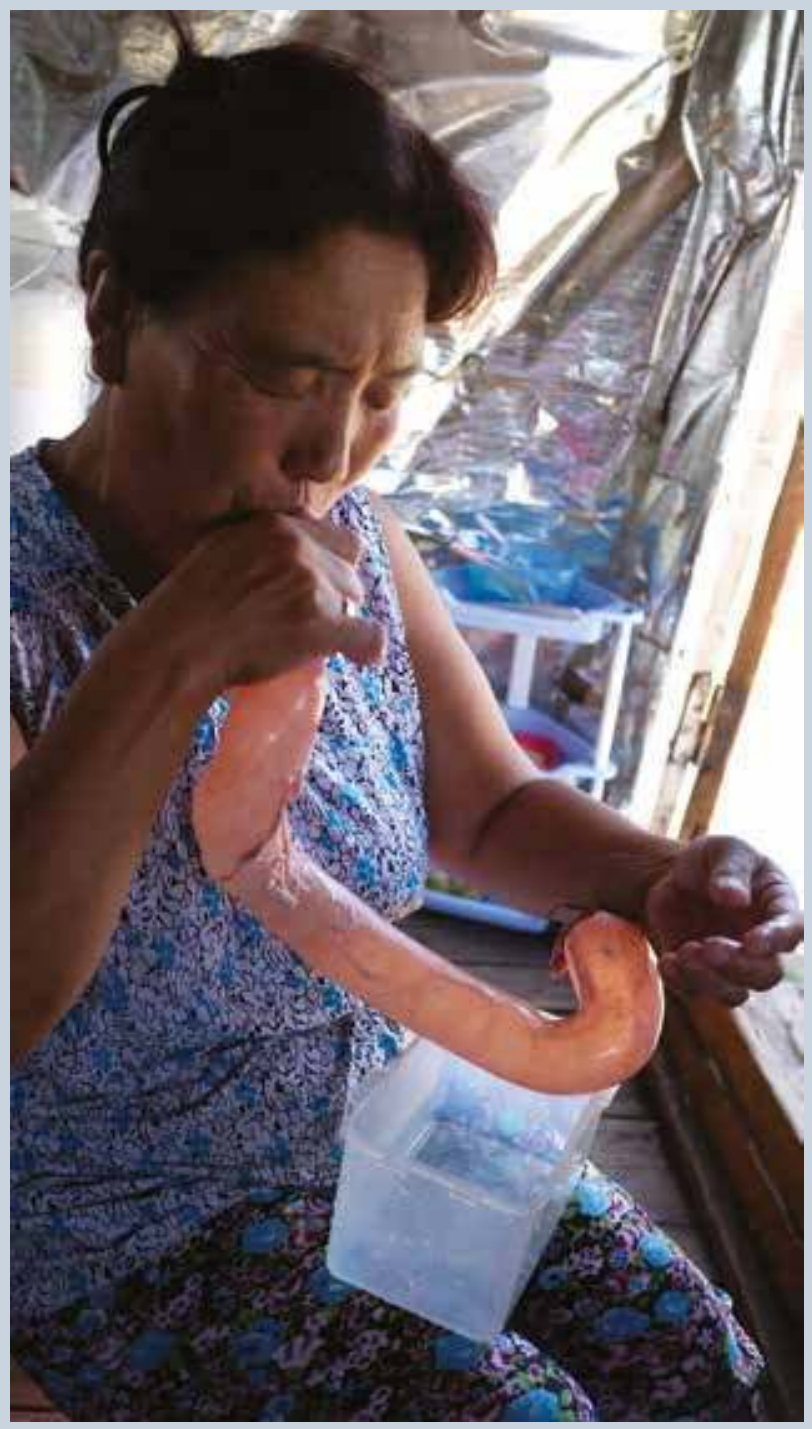

2. Elle lave le cæcum, puis le gonfle pour le faire sécher, enfin elle le dégonfle pour le remplir, tout en prenant garde à ce qu'il ne se rompe pas. Elle noue fermement un fil épais à l'une des extrémités puis, une fois le cæcum entièrement gonflé de l'air de ses poumons, ferme l'autre extrémité. Une fois gonflé d'air, elle ne force en rien la forme qu'il a prise, sous peine de l'endommager. Toute la difficulté est de ne pas percer le contenant ou de ne pas le faire éclater à chaque étape (lavage, gonflage, suspension pour le séchage, dégonflage, embossage).

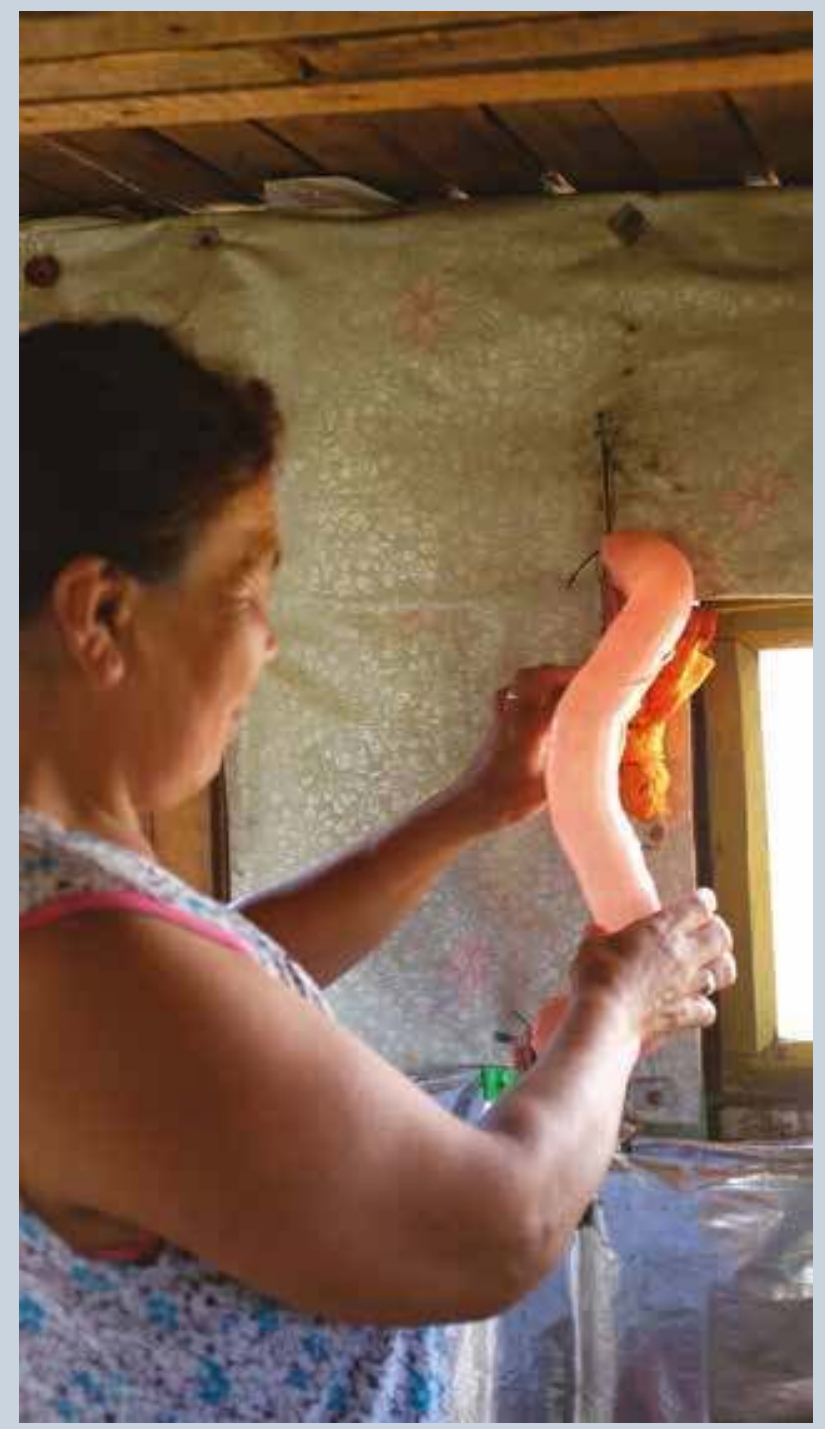

3. Selon la taille et l'épaisseur de la poche intestinale, le séchage complet dure de quelques heures à une journée entière.

Ce cæcum a séché en trois heures, à l'abri des mouches, dans un environnement sec et aéré, à une température ambiante avoisinant les $35^{\circ} \mathrm{C}$. Le bon séchage augure de la bonne ductilité du contenant une fois rempli de beurre mou, ou de beurre liquide tout juste fabriqué et versé encore chaud dans la poche. 
4. Ici, le lait de vache, «plus aigre que le lait de chèvre» mais aussi «plus gras et onctueux», a permis d'obtenir, une fois «longuement aéré et bien chauffé», une «peau crémeuse épaisse qui va bien fermenter». La femme a pu en tirer un «beurre bien consistant, jaune, avec un goût rance prononcé », qu'elle va légèrement faire fondre à feu moyen dans la marmite pour l'introduire dans le cæcum, dès qu'il aura fini de sécher.

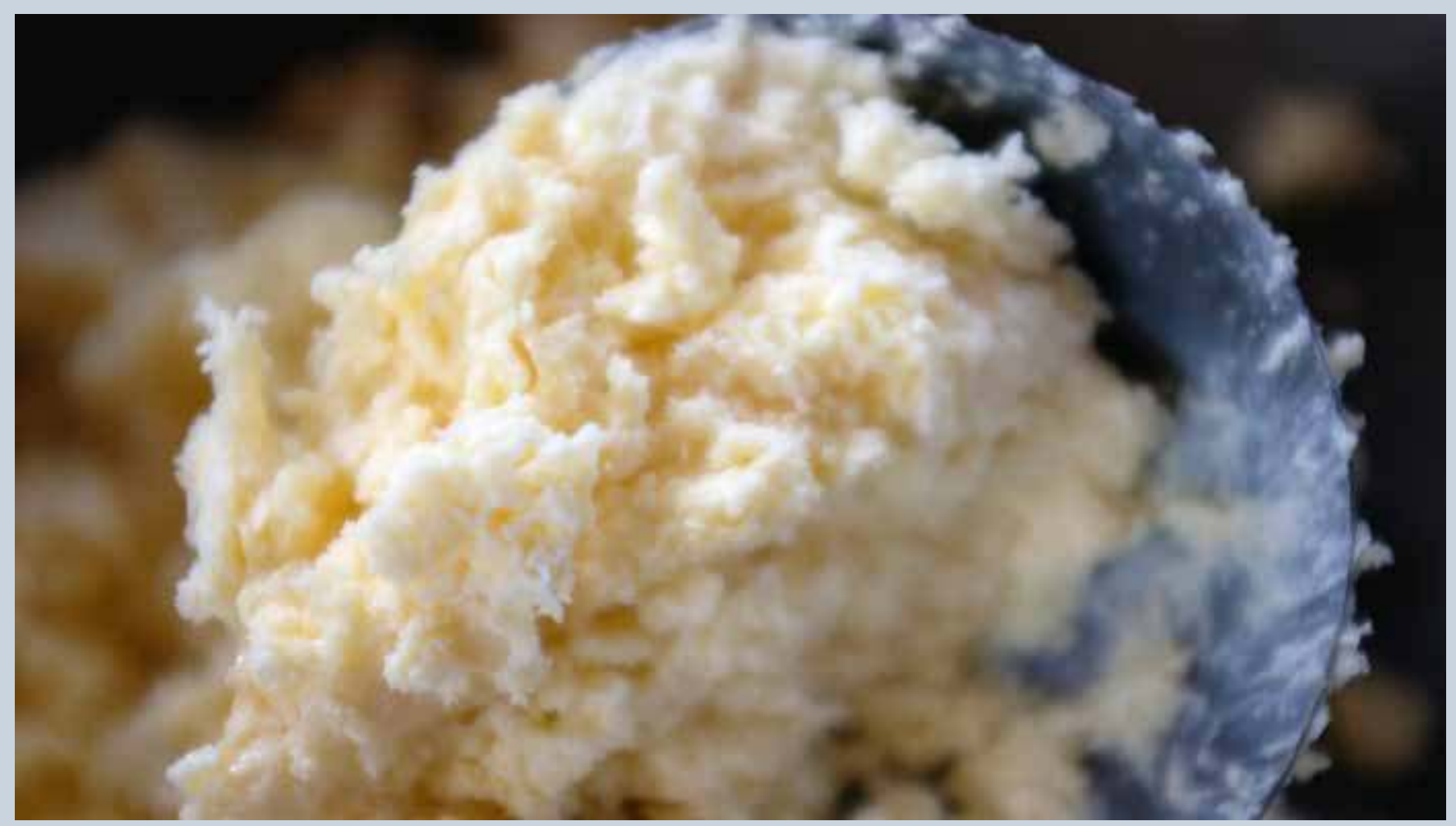



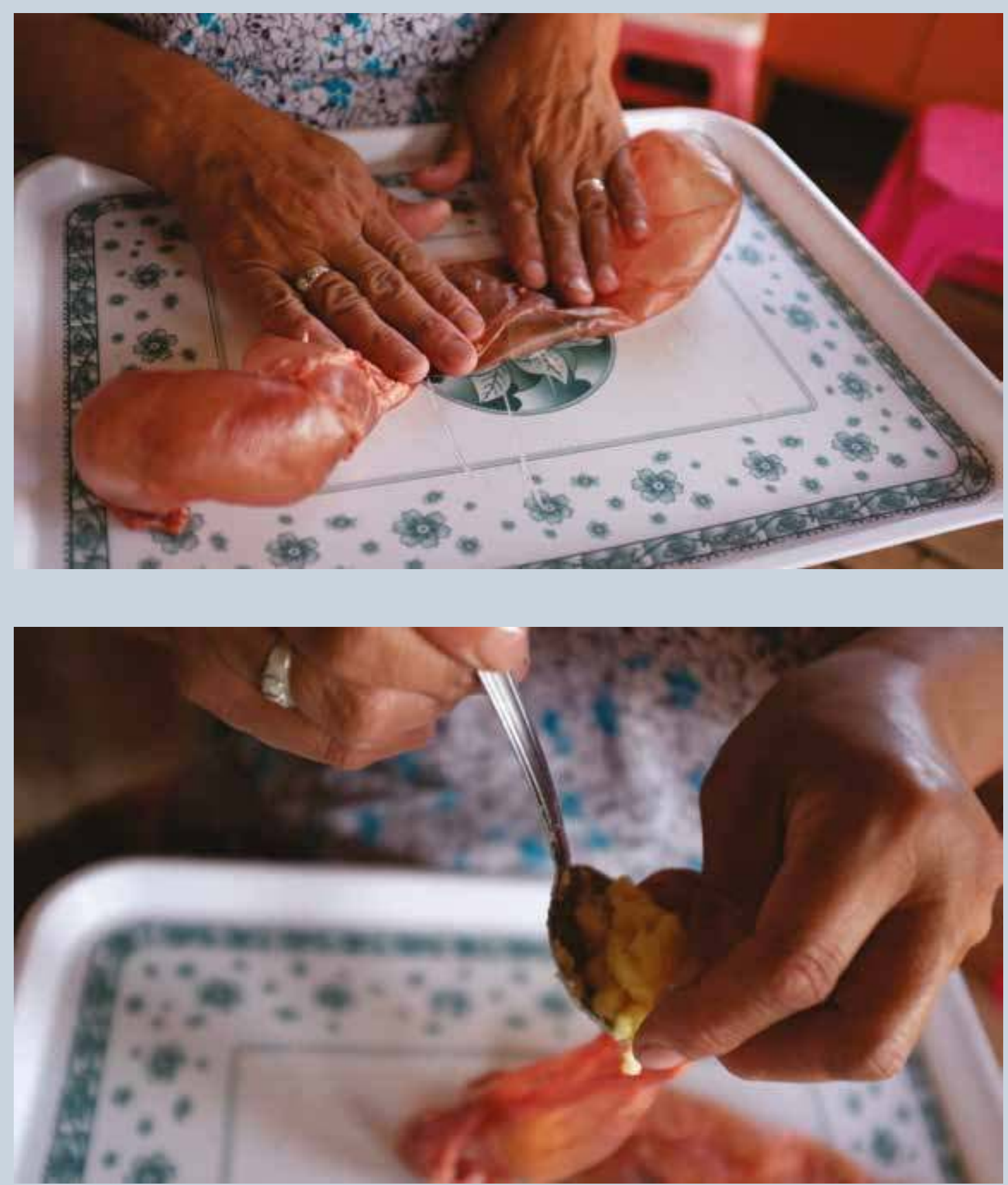

5. \& 6. L'embossage du cæcum est plus délicat que celui de la panse, dont la membrane est plus épaisse, solide, et l'ouverture beaucoup plus grande. Il s'agit d'introduire de petites quantités de beurre rance, ou de beurre composite?3, par l'extrémité choisie, puis de les faire glisser le long du tube, jusqu'à son autre extrémité, sans l'emplir totalement pour l'instant. L'embosseuse recourt à divers ustensiles pour mener à bien l'opération, qui lui prend sensiblement une demi-heure: outre les doigts de ses mains, qui maintiennent l'ouverture du cæcum en place puis font descendre lentement le beurre à l'intérieur, une petite cuiller pour introduire le beurre quand la paroi devient trop glissante, ou un entonnoir quand le beurre est liquide et chaud. Enfin, un support plat (plateau) pour stabiliser l'appendice dont la membrane retrouvant sa souplesse au contact de la graisse tient mal en place. 

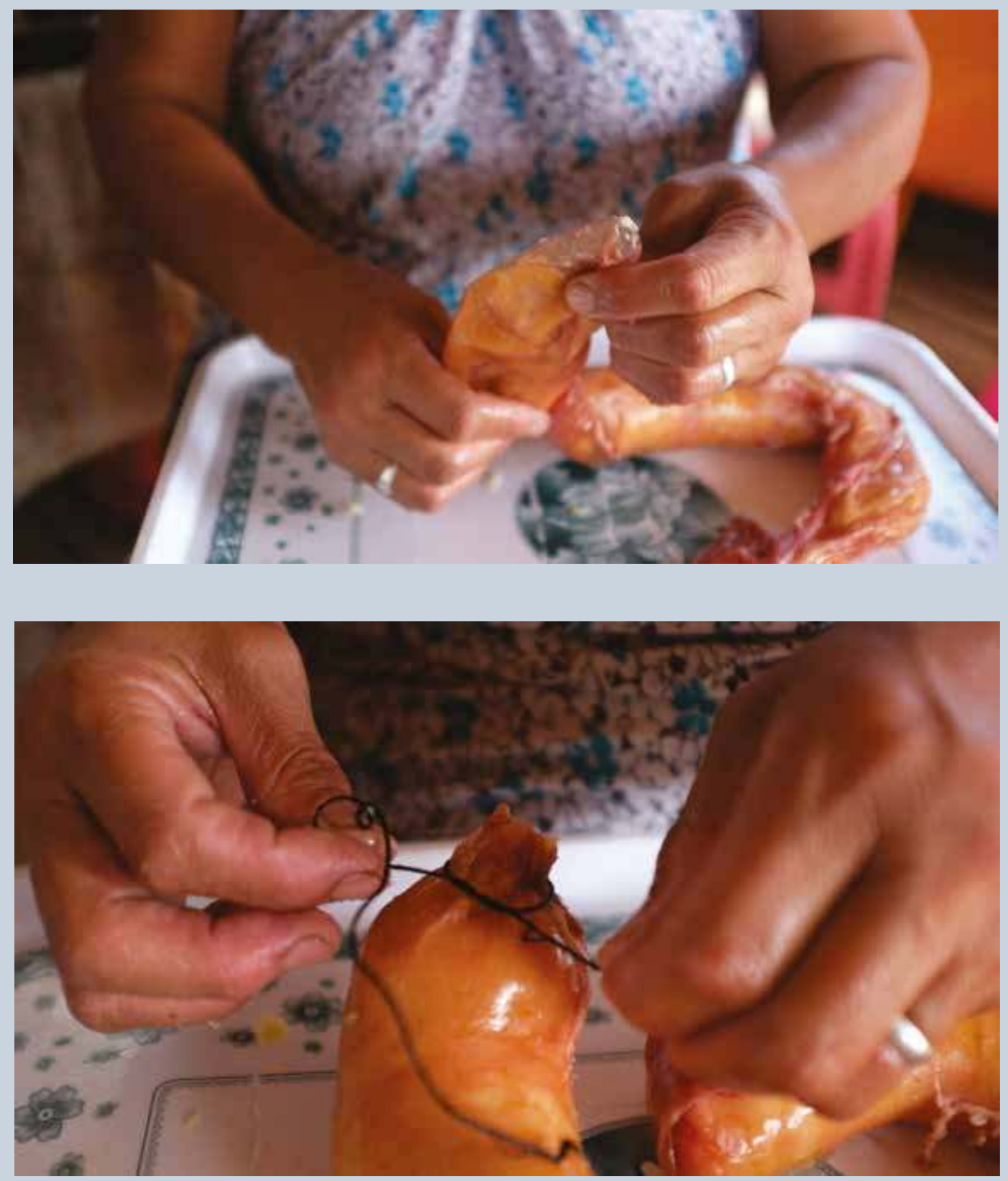

7. Pour supprimer toute particule d'air à l'intérieur de l'appendice cæcal, la dernière étape consiste à remplir le cæcum complètement, de manière que la femme ne puisse plus y introduire un milligramme de beurre. L'air n'altérerait pas la qualité du beurre, déjà rance, cependant il risquerait de provoquer l'ouverture du contenant et l'écoulement de beurre avant le premier prélèvement. Souillé par les poussières et saletés du sol de l'entrepôt de stockage, le beurre serait impropre à la consommation, constituant une «perte malheureuse». En guise de réparation, la maîtresse du foyer s'empresserait de «l'introduire dans la bouche du fourneau pour l'offrir à l'esprit-maître du feu», protecteur du foyer domestique.

8. La rigueur de l'embossage complet et de la bonne fermeture par ficelage assure l'hermétisme du contenant. 


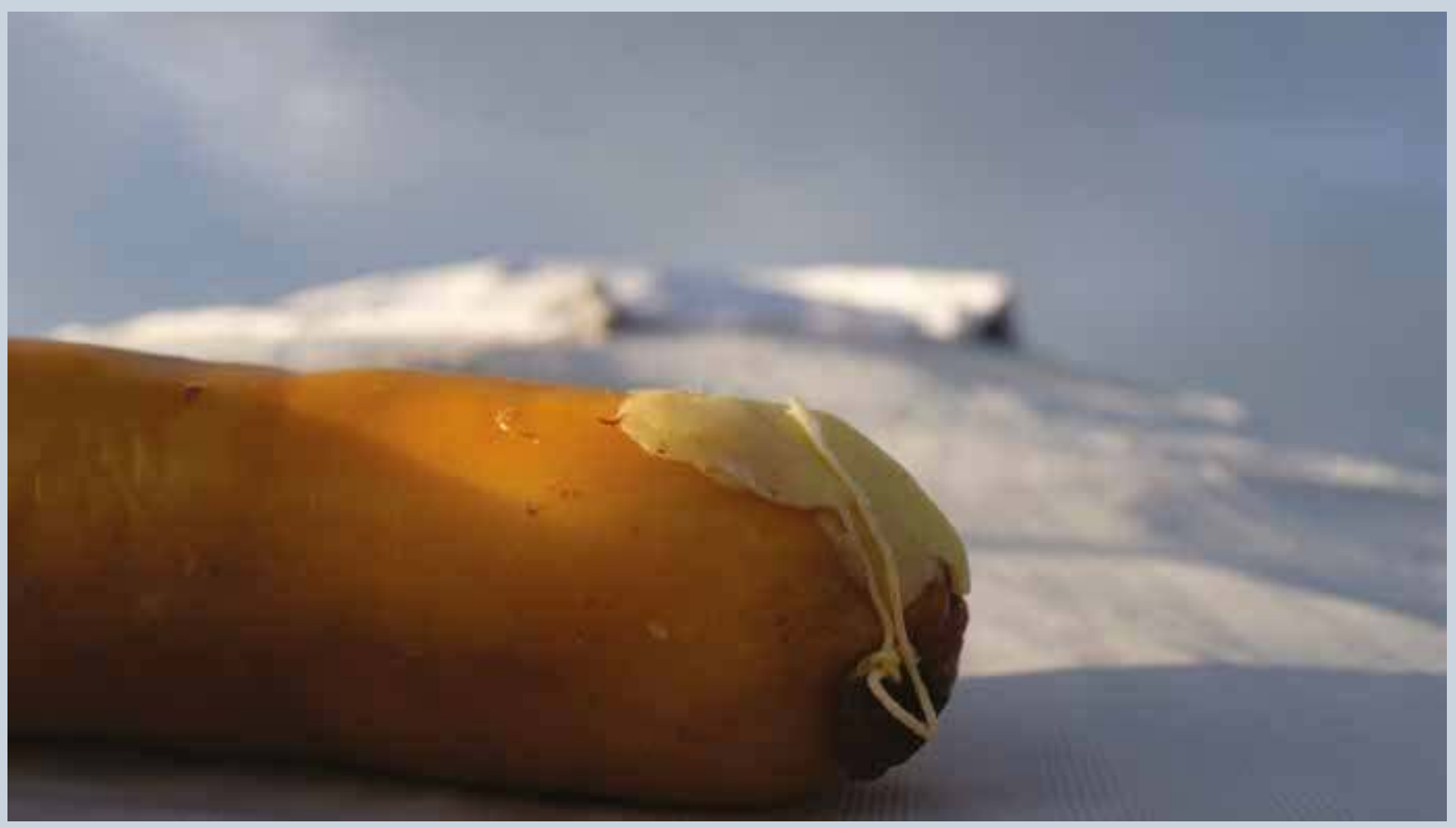

9. Voici un cæcum parfaitement obturé. Aucune bulle d'air ne provoquera l'ouverture de la membrane à l'arrivée brutale des températures hivernales quand le beurre se congèlera et se rigidifiera à leur contact. Le beurre rance apparent à son extrémité ne joue pas le rôle de cire pour le sceller; il illustre que les femmes n'essuient pas les panses et les boyaux une fois l'opération d'embossage terminée, comme nous essuierions un pot de confiture. 


\section{Chaîne opératoire (non exhaustive) de fabrication des crèmes et du beurre}

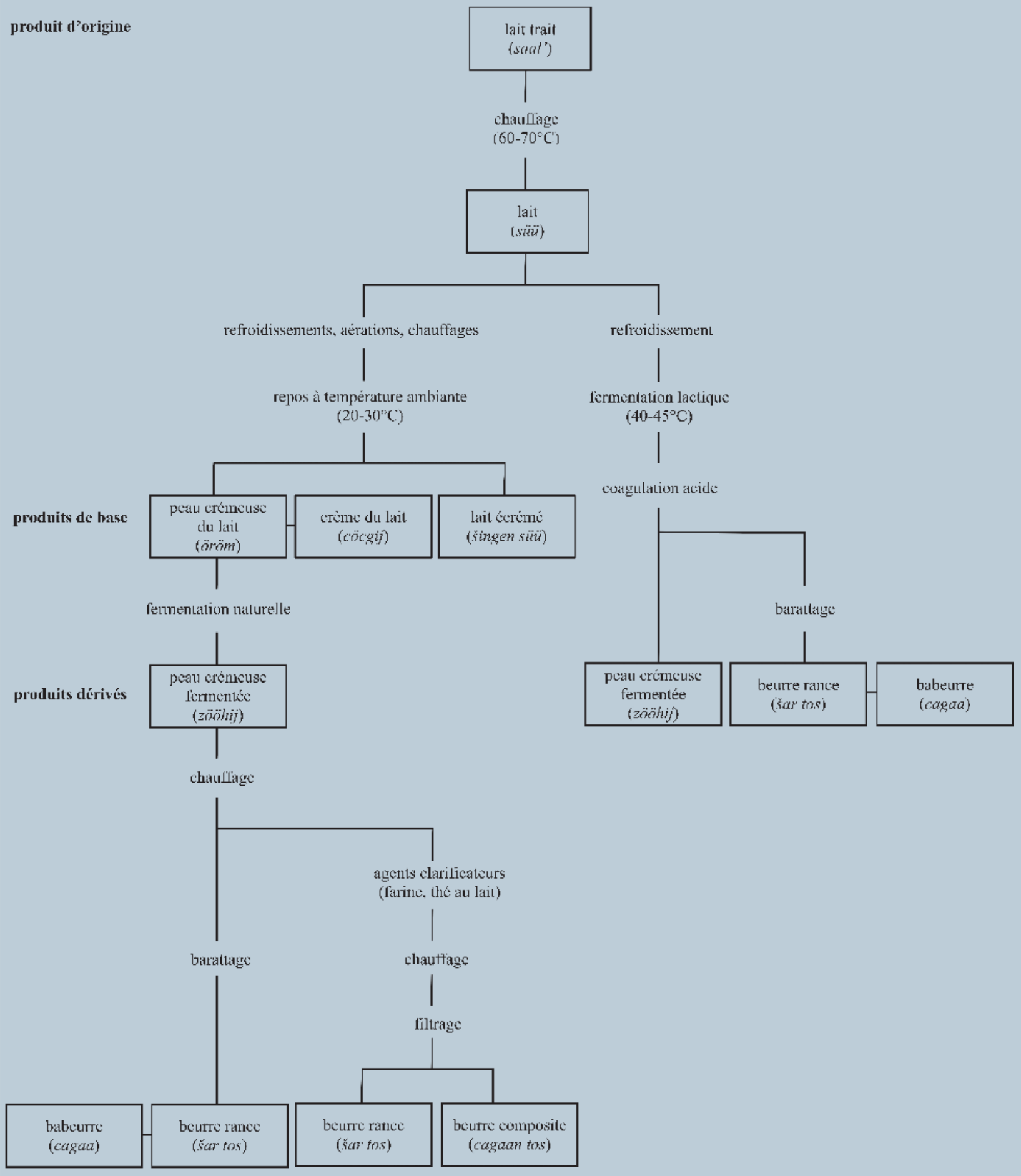


Chaîne opératoire (non exhaustive) de fabrication du yaourt et des fromages

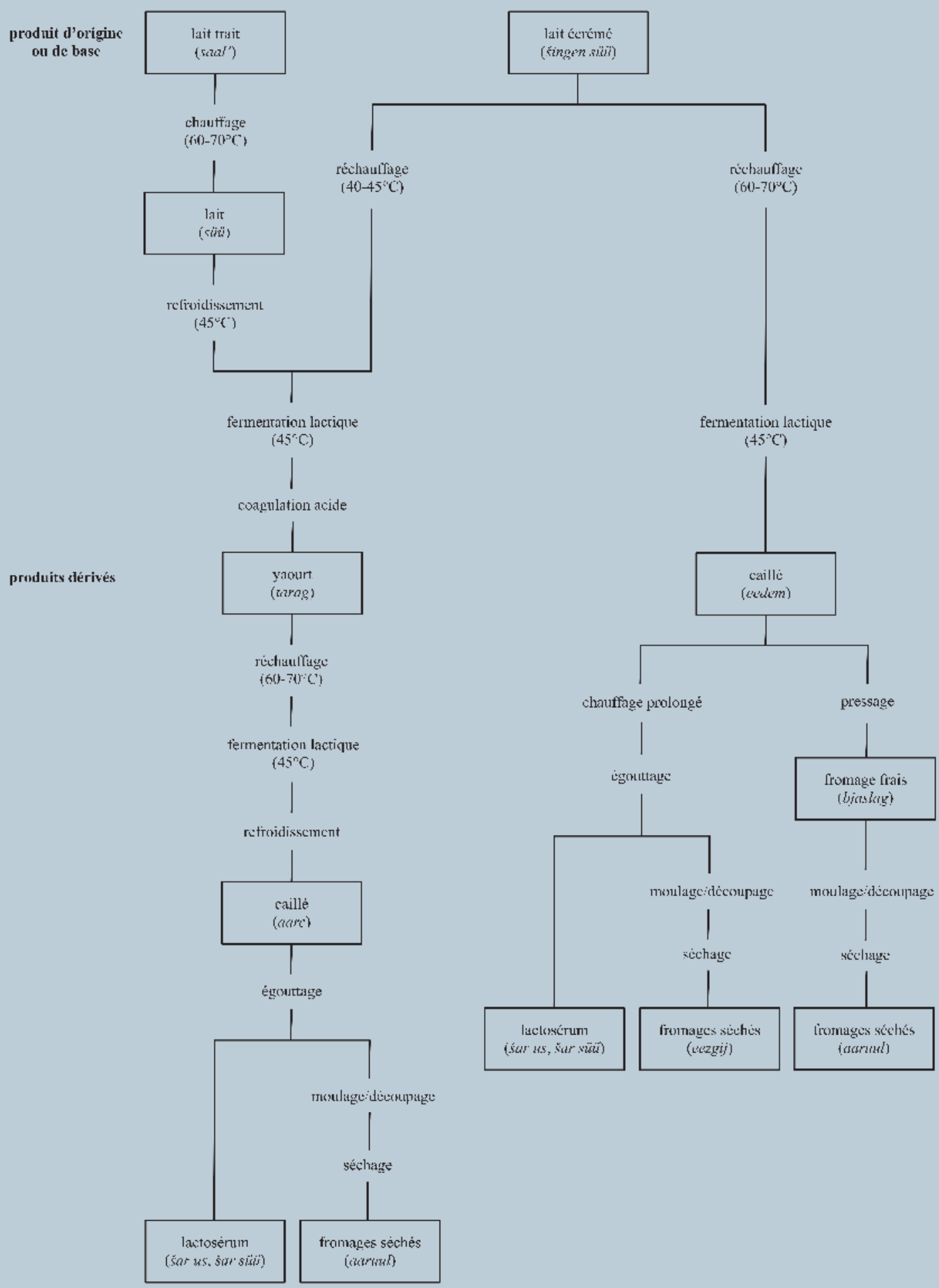


Plus les produits laitiers sont forts en bouche, en couleur et à l'odeur (rances, amers ou acides), plus ils sont estimés et socialement valorisés, c'est-à-dire à la fois «bons pour la santé, bien fabriqués, beaux pour l'âme». Ainsi privilégiés, les «aliments blancs» sont entassés sur les étals des marchés et supermarchés des capitales provinciales et d'Ulaanbaatar, et disparaissent plus rapidement que les laitages pasteurisés industriels. Comme les ruraux, les urbains ne craignent pas la rupture de la chaîne du froid: les coupures de courant électrique sont chroniques et les laitages frais n'en sont que plus appréciés quand le processus de fermentation reprend son cours.

Avec l'utilisation d'une matière animale comme contenant, rien ne se perd, tout se transforme. Le principe consiste à recycler ici un cæcum, là une panse. Multiples et successifs sont les usages des matières animales: le lait, la viande et la peau, sans compter les crins pour les cordes, la laine pour le feutre de revêtement de la yourte ou la doublure du manteau, les boyaux, le sang et les viscères pour faire des boudins, les os pour faire des protections, de la divination ou des jeux, les matières fécales séchées servant de combustible, etc. Divers sont les usages des poches et boyaux intestinaux: comme contenant pour stocker-transporter du beurre mais aussi du lait, de la peau crémeuse du lait, du yaourt, ou parfois des morceaux de chair, ou en qualité de récipient de cuisson pour cuire ensemble des morceaux de chair et/ou de viscères et du sang.

Les éleveurs nomades mongols stockent peu, mais stockent, ce qui rejoint la question $\mathrm{du}$ stockage en relation avec celle de la mobilité versus la sédentarité des sociétés, question chère à Alain Testart (1982a, 1982b, 1988) ${ }^{4}$. Léconomie de ces éleveurs reposant sur une production saisonnière et une mobilité relative, ponctuelle, le stockage relève ici davantage d'une «accumulation temporaire», pour reprendre l'expression de Tim Ingold (1983: 555) appliquée aux sociétés de chasseurs. Chez les éleveurs mongols, l'atténuation du flux des produits de l'élevage (lait, viande), marquée par une diminution des traites à destination des humains et la suspension de l'abattage pendant la longue période hivernale, induit un stockage limité des aliments. En période estivale d'affluence et de regroupement humain, l'accumulation des produits se régule par leur partage ou mise en circulation pour approvisionner les membres de la famille ou les marchés de la ville et de la capitale. 


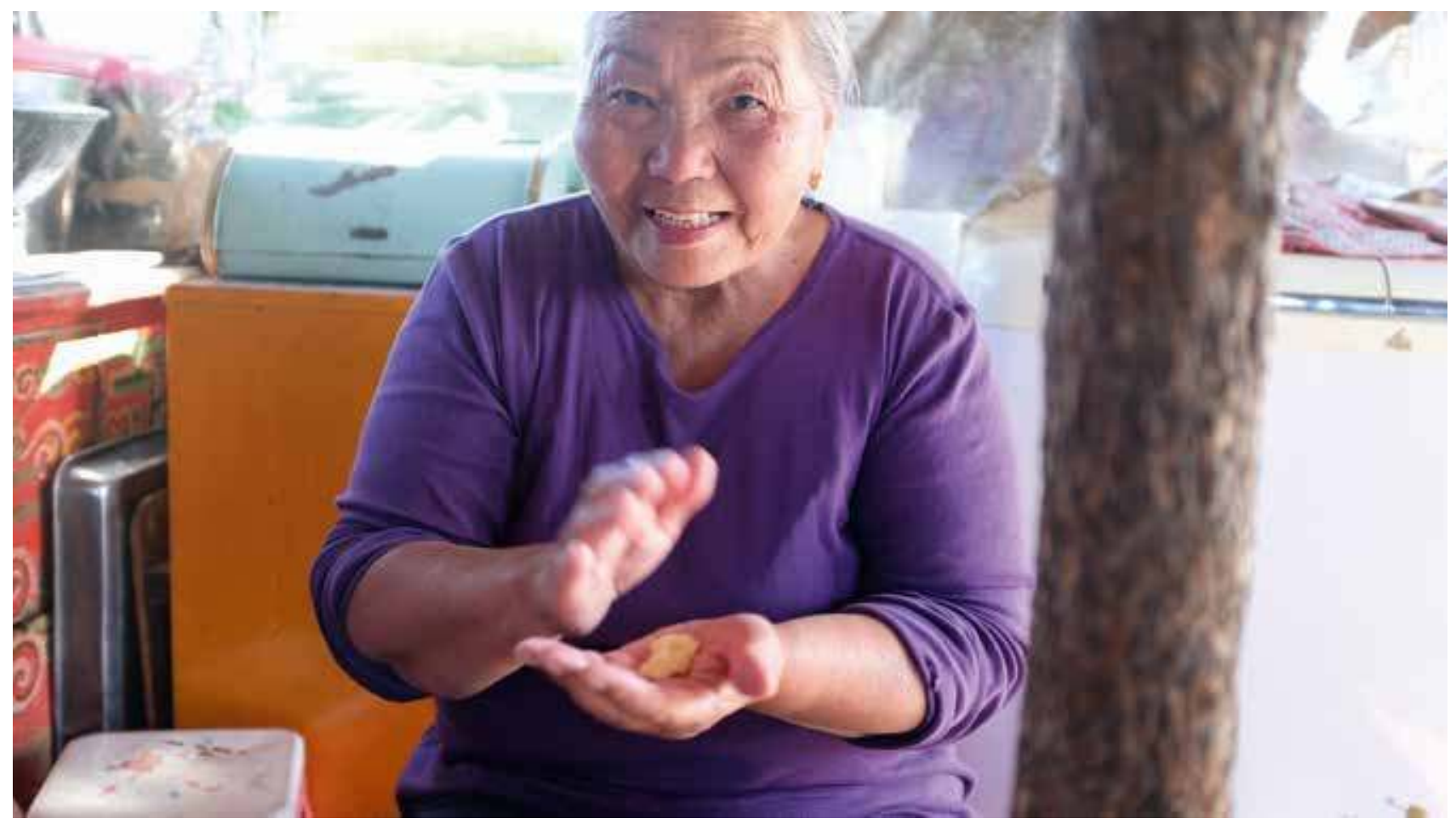

11. Sur les marchés de la capitale Ulaanbaatar, le beurre est vendu à la découpe, dans son contenant organique animal, aux côtés des autres laitages vendus au détail, les frais conditionnés sous cellophane, les séchés transportés dans un sachet plastique. Les urbains et ruraux prélèvent de petits morceaux de beurre qu'ils ajoutent dans la préparation du thé au lait ou celle d'une bouillie de yaourt et de riz, ou qu'ils dégustent, par gourmandise, sous forme de boulettes, comme me le montre fièrement cette citadine de passage dans la steppe.

- $\square$.

\section{Notes}

1. Qu'il soit limité ou intensif, le stockage est, dans certaines sociétés nomades, ici d'éleveurs, là de chasseurs-cueilleurs, un principe déterminant, plus déterminant que l'acquisition ou la conservation. Sur l'analyse des formes de sociétés et d'économies selon la présence/absence de stockage intensif, cf. Testart 1982a, 1982b.

2. De brebis, chèvre, chamelle, vache, yak ou hybride. Le chauffage prolongé initial (équivalent à une pasteurisation) transforme le produit de la traite (saal') en lait (süü), soit en un « aliment » (idee), précisément un «aliment blanc» (cagaan idee) (Ruhlmann 2015:
75). Je laisse de côté le lait de jument fermenté directement baratté dans une outre en peau de bœuf pour obtenir une boisson alcoolique.

3. Beurre mêlé de farine et de thé au lait, un produit reliquat du procédé de fabrication du beurre rance, de celui faisant intervenir des agents clarificateurs.

4. Et tant discutée entre autres par Tim Ingold (1983). Sur le débat qui les a longtemps animés, $c f$. notamment Testart 1982b, 1983, 1988, et Ingold 1983 et ses commentaires dans les articles de Testart, 1982b et 1988 . 


\section{I'auteure}

Sandrine Ruhlmann, chargée de recherche au CNRS (UMR 8173 Chine, Corée, Japon), travaille sur les pratiques alimentaires des Mongols Halh et s'intéresse à la question centrale du partage de nourriture, en lien avec le bonheur familial. Parallèlement, ses recherches portent sur la gestion des maladies animales en contexte postcommuniste. Elle mène actuellement une étude sur le processus de compromis alimentaire comme stratégie de résistance des peuples mongols sous domination (Mongolie, Mongolie méridionale en territoire chinois).

\section{Iconographie}

Image d'ouverture. La femme pose le cæcum rempli de beurre rance sur le toit de sa yourte, le temps de visiter une des autres yourtes du campement et de se lancer dans la préparation d'une nouvelle série de peaux crémeuses du

lait et de fromages. À la tombée de la nuit, elle pensera à la ranger dans l'entrepôt extérieur.

Crédits pour l'ensemble des images. ( ) S. Ruhlmann (Töv, été 2017).

\section{Références}

Ingold, T. 1983 «The Significance of Storage in Hunting Societies», Man 18(3) : 553-571.

Ruhlmann, S. 2015 L'appel du bonheur. Le partage alimentaire mongol. Paris: CEMS-EPHE.

Testart, A. 1982a Les chasseurs-cueilleurs ou l'origine des inégalités. Paris: Société d'Ethnographie.

— 1982b «The Significance of Food Storage Among Hunter-Gatherers: Residence Patterns, Population Densities, and Social Inequalities », Current Anthropology 23(5) : 523-537.

— 1988 «Some Major Problems in the Social Anthropology of Hunter-Gatherers », Current Anthropology 29 (1): $1-31$.

\section{Pour citer l'article}

Ruhlmann, S. 2018 «La seconde vie du cæcum ou le stockage ingénieux du beurre en Mongolie», Techniques\&Culture 69 «Le temps des aliments», p. 74-87. 